\title{
Coordinatrice en médecine ambulatoire
}

\author{
Bruno Gutknecht ${ }^{a}$, Ernst Gähler ${ }^{b}$ \\ a Avocat, Administration OrTra Formation professionnelle \\ b Vice-président de la FMH, responsable du département Professions paramédicales
}

Correspondance: Bruno Gutknecht odamed/OrTra Formation professionnelle Monbijoustrasse 35

CH-3011 Berne info[at]odamed.ch
Début octobre 2014, le Secrétariat d'Etat à la formation, à la recherche et à l'innovation (SEFRI) a publié dans la Feuille fédérale le règlement concernant l'examen professionnel de "coordinateur en médecine ambulatoire avec brevet fédéral/coordinatrice en médecine ambulatoire avec brevet fédéral». Cette fonction qui se décline en deux domaines différents, l'orientation clinique et l'orientation gestion, constitue une formation complémentaire pour les assistantes médicales (cf. www.odamed.ch pour de plus amples informations).

\section{Seule la Fédération suisse des associations d'assistantes médicales (FSAAM) a déposé un recours après la publication du règlement d'examen.}

La FMH a créé avec Médecins de famille Suisse, l'Association romande des assistantes médicales (ARAM), l'Association suisse alémanique des assistantes médicales (SVA) et l'Association suisse des écoles professionnelles médicales (SVMB) un organe responsable $\mathrm{du}$ projet et de l'examen: l'organisation du travail dénommée OrTra Formation professionnelle des assistantes médicales. Suite à de longues négociations avec les professions apparentées, OrTra Formation professionnelle des assistantes médicales a réussi à aplanir toutes les divergences au niveau des interfaces avec les profils professionnels (personnel soignant, service de soins à domicile, conseil en diététique, thérapies, etc.). Seule la Fédération suisse des associations d'assistantes médicales (FSAAM), qui regroupe trois associations d'assistantes médicales mineures de la région de Genève, du Tessin et de Suisse alémanique, a déposé un recours après la publication du règlement d'examen. Cette démarche est d'autant plus surprenante que la FSAAM avait assuré lors des nombreux entretiens qu'aucune opposition et en particulier aucun recours ne serait déposé au moment de la publication. Elle avait même demandé de le mentionner par écrit dans le procès-verbal d'une séance animée par l'OFSP.

Cette intervention de la FSAAM a pour conséquence de geler provisoirement toute la procédure d'approbation et de ce fait d'empêcher qu'un nombre important d'assistantes médicales qui se préparent au nouveau brevet - dont aussi des membres de la FSAAM - puissent se présenter comme prévu à la première session d'examen en 2015. Celles-ci courent par ailleurs le risque supplémentaire de voir expirer la validité d'une partie des certificats obtenus pour leurs modules avec un engagement personnel et financier important. Enfin, les négociations

tarifaires attendues depuis des années par le corps médical concernant la rémunération des prestations des assistantes médicales dans le cadre du masterplan pour la médecine de base ne peuvent être ni poursuivies ni menées à terme tant qu'aucun règlement d'examen n'a été approuvé avec un profil de compétences définitif.

Cette intervention de la FSAAM a pour conséquence de geler provisoirement toute la procédure d'approbation.

Le recours de la FSAAM ne présente aucune objection pertinente, que ce soit au niveau du contenu qu'au niveau du droit en matière de formation professionnelle. Elle s'oppose ainsi frontalement aux intérêts de tout le corps médical et d'une majeure partie des assistantes médicales de notre pays. Le SEFRI a ouvert la procédure de recours, il entendra les parties avant de trancher. La partie perdante aura ensuite la possibilité de se pourvoir devant le Tribunal administratif fédéral. 